\title{
The burden of co-existing dermatological disorders and their tendency of being overlooked among patients admitted to muhimbili national hospital in Dar es Salaam, Tanzania
}

Yassin M Mgonda* and Pauline NF Chale ${ }^{\dagger}$

\begin{abstract}
Background: Skin diseases are underestimated and overlooked by most clinicians despite being common in clinical practice. Many patients are hospitalized with co-existing dermatological conditions which may not be detected and managed by the attending physicians. The objective of this study was to determine the burden of co-existing and overlooked dermatological disorders among patients admitted to medical wards of Muhimbili National hospital in Dar es Salaam.
\end{abstract}

Study design and settings: A hospital-based descriptive cross-sectional study conducted at Muhimbili National hospital in Dar es Salaam, Tanzania.

Methods: Patients were consecutively recruited from the medical wards. Detailed interview to obtain clinicodemographic characteristics was followed by a complete physical examination. Dermatological diagnoses were made mainly clinically. Appropriate confirmatory laboratory investigations were performed where necessary. Data was analyzed using the 'Statistical Package for Social Sciences' (SPSS) program version 10.0. A p-value of < 0.5 was statistically significant.

Results: Three hundred and ninety patients admitted to medical wards were enrolled into the study of whom, $221(56.7 \%)$ were females. The mean age was $36.7 \pm 17.9$ (range 7-84 years). Overall, 232/390 patients (59.5\%) had co-existing dermatological disorders with 49\% (191/390) having one, 9\% (36/390) two and 5 patients (1\%) three. A wide range of co-existing skin diseases was encountered, the most diverse being non-infectious conditions which together accounted for 36.4\% (142/390) while infectious dermatoses accounted for 31.5\% (123/390). The leading infectious skin diseases were superficial fungal infections accounting for 18\%. Pruritic papular eruption of HIV/AIDS (PPE) and seborrheic eczema were the most common non-infectious conditions, each accounting for $4.3 \%$. Of the 232/390 patients with dermatological disorders, 191/232 (82.3\%) and 154/232 (66.3\%) had been overlooked by their referring and admitting doctors respectively.

Conclusion: Dermatological disorders are common among patients admitted to medical wards and many are not detected by their referring or admitting physicians. Basic dermatological education should be emphasized to improve knowledge and awareness among clinicians.

\footnotetext{
* Correspondence: ymgonda@muhas.ac.tz

† Contributed equally

Department of Internal Medicine, Muhimbili University of Health and Allied

Sciences, Dares Salaam, Tanzania
} 


\section{Background}

Skin diseases are diverse in clinical presentation, severity and epidemiology although dermatology may simply be considered by many as an outpatient service with very little attention given to admitted patients. Data from several studies on the overall community-based prevalence of skin diseases in developing countries indicate that they are quite common, ranging from $20 \%$ to $80 \%$ and most of them result from infections such as superficial mycoses, pyodermas and scabies [1-4]. Dermatological disorders affect all age groups of both sexes, however, children are more vulnerable and have been extensively studied worldwide [1,5-8]. The fact that dermatological disorders are very common in the general population and therefore, expected to be common even among hospitalized patients should not mean that they are of less medical importance than other systemic diseases.

There are, but relatively few studies which have documented on dermatological disorders in hospitalized patients [9-13]. The prevalence of dermatological disorders among admitted patients reported by some of these studies shows an almost similar spectrum, differing only in magnitude between developing and developed countries [14]. Conditions such as Stevens Johnson syndrome (SJS), toxic epidermal necrolysis (TEN), autoimmune blistering diseases, necrotizing fasciitis, leg ulcers, dermatological malignancies and psoriasis have been reported as the common indications for hospital admission in developing as well as the developed countries[14,15].

Reports from other studies rarely mention dermatological disorders as a primary reason for hospital admission. Most skin diseases may be overlooked by health professionals despite being important indicators of some underlying internal diseases. Skin diseases may coincidentally co-exist with other medical illnesses or be specific markers/manifestations of underlying systemic diseases. Some of the systemic diseases that can be suspected through cutaneous manifestations include chronic renal failure, endocrine disorders, lymphomas, nutritional deficiencies and HIV/AIDS. The HIV/AIDS pandemic has increased the number of hospital admissions worldwide, particularly in sub Saharan Africa and has been associated with a wide range of dermatological syndromes [16-18]. Cutaneous malignancies, candidiasis, dermatophytoses, molluscum contagiosum, seborrheic dermatitis and pruritic papular eruption have been frequently reported in individuals with HIV/AIDS, seen as inpatients or outpatients [19]. The objective of this study was therefore to describe the burden of co-existing dermatological disorders among patients admitted to medical wards of Muhimbili National hospital in Dar es Salaam and document the tendency if any, of being overlooked by their attending clinicians.

\section{Methods}

\section{Study design and setting}

This was a hospital-based cross sectional descriptive study conducted in the medical wards of Muhimbili National Hospital in Dar es Salaam, Tanzania. Muhimbili National Hospital is the largest tertiary referral and teaching hospital receiving patients from all regions and Dar es Salaam is the largest city in the country.

\section{Clinical work up}

Patients referred by medical officers (MD) from peripheral hospitals all over the country to Muhimbili national hospital's medical wards, were consecutively recruited into the study. Patient's files were systematically scrutinized to obtain useful demographic and clinical data. The referral dermatological diagnoses were retrieved from the patient's referral medical notes. Complete physical examination was conducted at admission by the admitting medical officer who made the 'dermatological diagnosis at admission'. These diagnoses were finally confirmed by one of the three dermatologists based in the hospital, and where necessary consultative discussions were held to make a common final diagnosis. The majority of the dermatological diagnoses were made clinically and where necessary and feasible, appropriate laboratory investigations were arranged.

\section{Laboratory procedures}

Appropriate specimens were collected and sent to the general pathology laboratory (since there is no specialized dermatology laboratory in the hospital) for bacteriological, mycological, parasitological or histopathological studies as appropriate. Specimens from suspected fungal lesions underwent microscopic examination after treatment with $10 \%$ potassium hydroxide solution. Skin scrapings from scabies lesions were obtained from burrows and examined for mites under a light microscope with a drop of normal saline. Skin biopsy specimens were placed in bottles containing $10 \%$ buffered formalin before being stained with Haematoxylin and Eosin and then mounted on Dextrene Polystyrene Xylene (DPX), followed by microscopic examination.

\section{Data analysis}

Data analysis was done using statistical package for social sciences (SPSS) computer program version 10.0. $\mathrm{P}$-value less than 0.05 was regarded as significant.

\section{Ethical considerations}

Ethical clearance was obtained from the ethical clearance board of the Muhimbili University of Health and Allied Sciences (MUHAS). Permission to conduct the 
study in the medical wards of Muhimbili National Hospital $(\mathrm{MNH})$ was sought from the hospital authority. Fully informed verbal and written consent was obtained from every patient who participated in the study.

\section{Results}

A total of 390 patients admitted to medical wards were recruited into the study. The demographic characteristics and prevalence of dermatological disorders among these patients has been shown in table 1 . The mean age was 36.7 years $(\mathrm{SD} \pm 17.9)$ with the majority $(52.3 \%)$ being young adults aged $18-44$ years and 56.7\% (221/390 were females. Overall, 232/390 patients (59.5\%) had co-existing dermatological disorders with 49\% (191/390) having one, $9 \%(36 / 390)$ two and 5 patients (1\%) three. Patients in age groups $18-31$ years (70\%) and $32-44$ years $(66 \%)$ had the highest prevalence than the other age groups $(\mathrm{p}=$ 0.01 and 0.04 respectively).

Table 2 shows the dermatological disorders among patients admitted to medical wards. A wide range of skin diseases was encountered, the most diverse being noninfectious conditions which put together accounted for $36.4 \%$ (142/390), while infectious dermatoses accounted for $31.5 \%$ (123/390). The common non infectious skin diseases were pruritic papular eruption of HIV/AIDS (PPE) present in $4.3 \%$ (17/390), seborrheic dermatitis in 3.8\% (15/390), non specific ulcers in 3.8\% (15/390) followed by acne vulgaris and keloids, all present in 3\%. The common infectious dermatoses were superficial fungal infections at a prevalence of $18 \%$ (70/390) with dermatophytoses (tinea) accounting for $10 \%$, pityriasis versicolor $4.6 \%$ and oral thrush 3.3\%. The commonest dermatophytoses were tinea corporis and tinea unguium both with prevalence of 3.6\%. Bacterial skin infections (pyodermas) were seen in 7\% (28/ 390 ), viral infections in $5 \%$ and 4 patients (1\%) had scabies. Kaposi's sarcoma was the commonest malignancy observed in $11 / 390(2.8 \%)$ of the 12 patients with cutaneous neoplasms. One patient had squamous cell carcinoma.
Table 3 shows the pattern of dermatological disorders as detected at pre-admission, admission and post-admission periods. Out of 390 admitted patients, 41(10.5\%) were reported as having dermatological disorders preadmission, with Kaposi's sarcoma being over diagnosed in 3 patients giving a false prevalence of $3.6 \%$ against the actual prevalence of $2.8 \%$. At admission, $20 \%$ were reported as having dermatological disorders, the leading diagnoses being fungal infection in $5.1 \%$. Kaposi's sarcoma was reported in 15 patients (3.8\%) being an over diagnosis by 4 patients. In the post-admission period when patients were thoroughly reviewed and dermatological diagnoses established, confirmed or ruled out, a total of 232/390 (59.5\%) patients were found to have dermatological disorders. In general, of the $232 / 390$ patients with dermatological disorders, 191/232 (82.3\%) and 154/ 232 (66.3\%) had been overlooked by the referring and admitting doctors respectively.

Table 4 shows the pattern of co-existence between dermatological disorders and other medical diseases. Overall, $133 / 390$ (34.1\%) patients were admitted due to infectious diseases and of these, $82 \%(109 / 133)$ had co-existing dermatological disorders. The infectious diseases which had co-existing dermatological disorders were: HIV disease (86/109; 79\%), tuberculosis (13/109; 12\%), malaria (7/109; $6.4 \%)$, meningitis, $(2 / 109 ; 2 \%)$ and pneumonia $(1 / 109 ; 1 \%)$. Dermatological disorders which mostly co-existed with HIV disease included Kaposi's sarcoma (11/11; 100\%), PPE $(15 / 17 ; 88.2 \%)$, oral candidiasis $(11 / 13 ; 8.6 \%)$, psoriasis $(4 / 5 ; 80 \%)$ common warts $(10 / 13 ; 77 \%)$, and seborrheic dermatitis $(10 / 15 ; 66.7 \%)$.

Thirty three patients were admitted due diabetes mellitus and 22 of them (66.7\%) had co-existing dermatological disorders commonly tinea lesions $45.4 \%$ (10/22) and ulcers $18.1 \%$ (4/22). Among 75/390 (19.2\%) patients with cardiovascular diseases, 33 (44\%) had co-existing dermatological disorders. Fifty five patients (14\%) were admitted due to hematological diseases and $43.6 \%(24 / 55)$ had co-existing

Table 1 The prevalence of dermatological disorders among patients admitted to medical wards distributed by age and sex $(n=390)$

\begin{tabular}{llll}
\hline Variable & All patients $\mathbf{n}(\%)$ & Patients with dermatological disorder $\mathbf{n}(\%)$ & OR (95\% Cl \\
\hline Sex & $221(56.7)$ & $122(55.2)$ & 1.0 \\
Female & $169(43.3)$ & $110(65.1)$ & $1.51(0.99-2.29)$ \\
Male & & & 0.05 \\
& & $29(49.2)$ & 1.0 \\
Age (years) & $59(15.1)$ & $73(69.5)$ & $2.36(1.20-4.63)$ \\
$\mathbf{7 - 1 7}$ & $105(26.9)$ & $65(65.7)$ & $1.98(1.01-3.86)$ \\
$\mathbf{1 8 - 3 1}$ & $99(25.4)$ & $41(51.3)$ & 0.01 \\
$\mathbf{3 2 - 4 4}$ & $80(20.5)$ & $24(51.1)$ & $1.09(0.55-2.14)$ \\
$\mathbf{4 5 - 6 0}$ & $47(12.1)$ & $\mathbf{2 3 2 ( 5 9 . 5 )}$ & 0.81 \\
$\mathbf{6 1 - 8 4}$ & $\mathbf{3 9 0 ( 1 0 0 )}$ & 0.85 & $-1.08(0.49-2.33)$ \\
Total & & & -
\end{tabular}


Table 2 Dermatological disorders among patients admitted to medical wards $(n=390)$

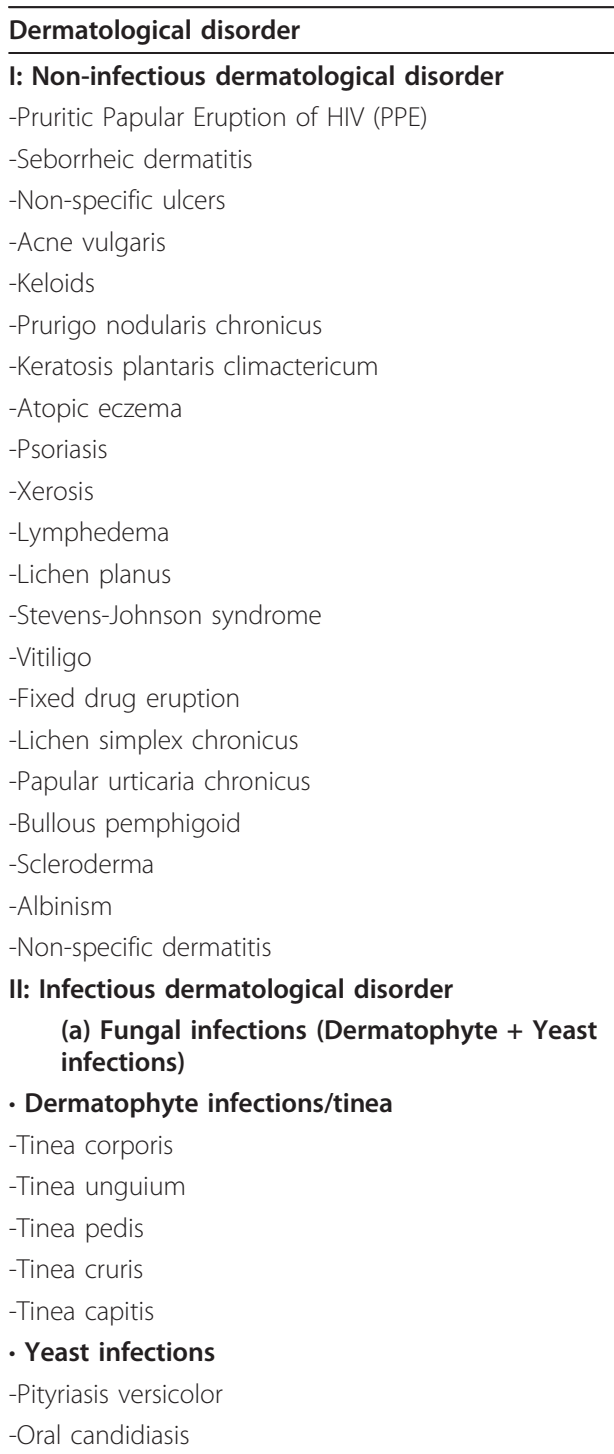

(b) Bacterial infections

-Infected ulcers

-Erythrasma

-Cellulitis

-Furuncles/carbuncles

-Superficial folliculitis

-Impetigo

(c) Viral infections

-Common warts

-Herpes simplex

-Genital warts

-Chicken pox

-Molluscum contagiosum

-Herpes zoster

(d) Parasitic infestations

-scabies

III: Neoplatic dermatological disorders
Table 2 Dermatological disorders among patients admitted to medical wards $(n=390)$ (Continued)

\begin{tabular}{ll}
\hline -Kaposi's sarcoma & $11(2.8)$ \\
-Squamous cell carcinoma & $1(0.2)$ \\
Total & $\mathbf{2 3 2 ( 5 9 . 5 )}$ \\
\hline
\end{tabular}

dermatological disorders: leukemia $(9 / 24 ; 37.5 \%)$, sickle cell disease $(9 / 24 ; 37.5)$ and anemia $(6 / 24 ; 25 \%)$. Among 29 (7.4\%) patients with renal diseases, 16 (55.1\%) had coexisting dermatological disorders while of the 19 patients (4.9\%) admitted due to liver diseases, 12 (63.1\%) had coexisting dermatological disorders, mostly xerosis generalisata and prurigo simplex. Twenty six patients (6.7\%) were admitted due to solid malignancies and of these, 12 (46.1\%) had co-existing dermatological disorders.

\section{Discussion}

It may commonly been remarked that dermatology is usually considered as an outpatient specialty associated with low mortality [15]. This notion could lead to less dermatological attention given to hospitalized patients by some of their attending physicians. Less dermatologic attention paid to admitted patients may allow most of the skin diseases to run a chronic course with significant effects on the general health as well as the quality of life of the affected individual.

Certain systemic disorders can be suspected through cutaneous symptoms and signs. This hospital-based cross sectional study has described the magnitude of coexisting dermatological disorders among patients admitted to medical wards of a national consultant hospital. Almost all forms of skin diseases (infectious, noninfectious, neoplastic, non-specific rashes) were encountered although at different frequencies. When specific types of dermatological disorders were analyzed, the most common were fungal infections $(18 \%)$, bacterial infections (7.1\%), and viral infections (5.3\%). Many community based studies conducted in developing tropical countries have described infectious dermatological disorders, especially fungal and bacterial infections as being the most commonly encountered [1-3,11].

The pattern of co-existence between dermatological and other medical conditions in our study demonstrated that, over three-quarters of patients with PPE, seborrheic dermatitis, oral candidiasis, cutaneous warts and psoriasis had HIV/AIDS. On the other hand, all patients with Kaposi's sarcoma $(11 / 11 ; 100 \%)$ were HIV infected. HIV infection has been reported to cause its greatest impact on the skin whereby, today over fifty different types of HIV-related skin diseases have been documented [14-16]. Various studies on HIV related mucocutaneous manifestations conducted worldwide, have documented HIV related skin diseases as being very 
Table 3 Dermatological disorders diagnosed at pre admission, admission and post admission periods among patients admitted to medical wards $(n=390)$

\begin{tabular}{|c|c|c|c|}
\hline \multirow[b]{2}{*}{ Dermatological disorder } & \multicolumn{3}{|c|}{ Time of diagnosis } \\
\hline & Pre-admission $\mathrm{n}(\%)$ & Admission $\mathrm{n}(\%)$ & Post-admission $\mathrm{n}(\%)$ \\
\hline All types & $41(10.5)$ & $78(20)$ & $232(59.5)$ \\
\hline I: Non-Infectious disorders & $36(9.2)$ & $52(13.3)$ & $142(36.4)$ \\
\hline -Inflammatory dermatoses* & $12(5.6)$ & $23(3.8)$ & $58(15)$ \\
\hline -Kaposi's sarcoma & $14(3.6)$ & $15(3.8)$ & $11(3)$ \\
\hline -Squamous cell carcinoma & 0 & 0 & $1(0.3)$ \\
\hline -Nonspecific lesions & $10(2.6)$ & $14(3.6)$ & $72(18.2)$ \\
\hline II: Infectious disorders & $5(1.3)$ & $35(9)$ & $123(31.5)$ \\
\hline -Fungal infections & $2(0.5)$ & $20(5.1)$ & $70(18)$ \\
\hline -Bacterial infections & $2(0.5)$ & $8(2.0)$ & $28(7.1)$ \\
\hline -Viral infections & $1(0.2)$ & $7(1.8)$ & $21(5.3)$ \\
\hline -Mite Infestation & 0 & 0 & $4(1.0)$ \\
\hline - (Scabies) & & & \\
\hline
\end{tabular}

common [14-17]. In this study it has also been observed that, a wide variety of systemic diseases co-existed with dermatological conditions. Systemic diseases which demonstrated high frequency of dermatological disorders $(>50 \%)$ included diabetes mellitus, chronic kidney disease, hematological disorders hepatic diseases and neurological diseases.

The finding of cellulitis in only $1.5 \%$ of admitted patients in our study, while cellulitis tends to be one of the frequent causes of admission in developed countries may not be surprising since in our set up, mild forms of cellulitis would normally be managed at peripheral hospitals while severe cellulitis which is usually associated with dermal necrosis and fasciitis would be admitted to surgical (and not medical) wards for surgical interventions such as surgical toilet, slouphectomy and even skin grafting.

The prevalence of dermatological disorders at preadmission (referral) and admission periods, was grossly underestimated for all disorders except for Kaposi's sarcoma which was over-diagnosed. Most skin diseases were overlooked by the referring and admitting doctors.
In many areas, health professionals have been reported as being unaware of the burden of dermatological diseases [5]. Health workers tend to overlook or even ignore skin diseases despite the fact that some of them form important signs or symptoms of the underlying internal diseases. This apparent 'unawareness' attitude has been partly attributed to inadequate knowledge of dermatological disorders among clinicians [5]. On the other hand, it could also be speculated that the majority of patients presenting to hospitals for various diseases, may not complain about their accompanying dermatological problems (which would have enabled clinicians to easily detect them), probably due to the assumption that skin diseases are a mere cosmetic nuisance, not associated with any serious suffering. All these factors may lead to delays in diagnosing the underlying serious and even life threatening systemic diseases.

\section{Conclusion}

Dermatological disorders are very common and diverse among patients admitted to medical wards but they are rarely documented as primary or additional diagnoses

Table 4 The frequency of co-existence of dermatological disorders with systemic diseases among patients admitted to medical wards $(n=390)$

\begin{tabular}{lll}
\hline Systemic disease & Frequency among Admitted patients $\mathbf{n}(\%)$ & Frequency of co-existing dermatological disorders $\mathbf{n}(\%)$ \\
\hline Infectious diseases & $133(34.1)$ & $109(82)$ \\
Cardiovascular diseases & $75(19.2)$ & $33(44)$ \\
Hematological diseases & $55(14.1)$ & $24(43.6)$ \\
Diabetes mellitus & $33(8.5)$ & $22(66.7)$ \\
Renal diseases & $29(7.4)$ & $19(55.1)$ \\
Hepatic diseases & $19(4.9)$ & $12(63.1)$ \\
Solid malignancies & $26(6.7)$ & $12(46.1)$ \\
Neurological diseases & $14(3.6)$ & $8(57.1)$ \\
\hline
\end{tabular}


by their referring or admitting physicians. Dermatological education should be emphasized to the health care workers to improve their knowledge.

\section{Study limitation}

Lack of a specialized dermatology laboratory led to failure to make some confirmatory tests like virological identification of viral infections, immunofluorescent microscopy for blistering diseases and antinuclear antibody tests for connective tissues disorders.

\section{Acknowledgements}

The authors would like to thank the Ministry of Health and Social Welfare and the Muhimbili University of Health and Allied Sciences for the financial and other logistical supports; Muhimbili National Hospital allowed this study to be conducted in its medical wards. Dr Candida Moshiro of the department of epidemiology and statistics offered great statistical assistance. Finally the authors thank all parents in the medical wards for their co operation and willingness to participate in the study.

\section{Authors' contributions}

YMM conceived the study, supervised data collection and prepared the final manuscript as a corresponding author. PNFC participated in study design, conducted data collection and prepared the preliminary manuscript. All authors read and approved the final manuscript.

\section{Competing interests}

The authors declare that they have no competing interests.

Received: 26 October 2010 Accepted: 14 April 2011

Published: 14 April 2011

\section{References}

1. Ferié J, Dinkela A, Mbata M, et al: Skin disorders among school children in rural Tanzania and an assessment of therapeutic needs. Tropical Doct 2006, 36(4):219-221.

2. Walker SL, Shah M, Hubbard VG, et al: Skin disease is common in Rural Nepal; result of point prevalence study. British Journal of Dermatology 2008, 158(2):34-338.

3. Abdel HK, Abdel A, Hofny ERM: Prevalence of skin diseases in rural areas of Assiut Governorat, Upper Egypt. Int J Dermatol 2003, 24(11):887-892.

4. Gibbs S: Skin disease and socioeconomic conditions in rural Africa: Tanzania. Int J Dermatol 1996, 35(9):633-9.

5. Wilfried S: Community health workers reduce skin diseases in East African children. Int J Dermatol 2002, 37(5):370-377.

6. Mgonda YM, Lutale JJ: Acne in secondary school students in Dar es Salaam: perceptions, attitudes and implications. Tanzania Medical Journal 2001, 16(2):21-4

7. Robbert SA, Johannes C, Sander K, et al: Association between skin diseases and severe bacterial infections in children: case-control study. BMC Family Practice 2006, 7:52.

8. Masawe AEJ, Nsanzumuhire $H$, Mhalu F: Bacterial skin infections in preschool and school children in coastal Tanzania. Arch Dermatol 1975, 111:1312-6.

9. Figueroa JI, Fuller LC, Abrah A, et al: Dermatology in the southernwestern Ethiopia: rational for a community approach. Int J Dermatol 1998 37:752-8.

10. Mahreen A, Beverley A: Community Dermatology. J Comm Dermatol 2006, 3(4):17-32.

11. Henderson CA: Skin disease in rural Tanzania. Dermatol Practice 1986, 4:14-19.

12. Souissi A, Zeglaoui F, Zouari B: Skin Disease in Tunisia. Epidemiological Report. Acta Dermatoven APA 2007, 16(3):111-116.

13. Satimia FT, McBridge SR, Leppard B: Prevalence of skin disease in rural Tanzania and factors influencing the choice of health Care, Modern or Traditional. Arch Dermatology 1998, 134(9):1363-1366.
14. Jessop SF, Mckenzie RF, Milne J, et al: Pattern of admissions to a tertiary dermatology unit in South Africa. Int J Dermatol 2002, 41(9):568-570.

15. George MCSusannah, Harrison ADavid, Welch ACatherine, Nolan MKathleen, Friedmann SPeter: Dermatological conditions in intensive care: a secondary analysis of the Intensive Care National Audit \& Research Centre (ICNARC) Case Mix Programme Database. Critical Care 2008, 12:S1.

16. Maurer Toby A: Dermatological manifestations of HIV Infection. International AIDS Society 2005, 13(5):149-153.

17. Thompson DS, Bain B, Innis AE: The prevalence of Mucocuteneous Disorders among HIV-positive patients attending an Outpatients Clinic in Kingston, Jamaica. West Indian Med J 2008, 57(1):54.

18. Jack SR, Marta VB, Lisa F, et al: Etiology of Pruritic Papular Eruption with HIV Infection in Uganda. JAMA 2004, 292(21):2614-2621.

19. Goodman DS, Teplitz ED, Wishner A, et al: Prevalence of cutaneous disease in patients with acquired immunodeficiency syndrome (AIDS) or AIDS-related complex. J Am Acad Dermatol 1987, 17(2 Pt 1):210-20.

Pre-publication history

The pre-publication history for this paper can be accessed here: http://www.biomedcentral.com/1471-5945/11/8/prepub

doi:10.1186/1471-5945-11-8

Cite this article as: Mgonda and Chale: The burden of co-existing dermatological disorders and their tendency of being overlooked among patients admitted to muhimbili national hospital in Dar es Salaam, Tanzania. BMC Dermatology 2011 11:8.

\section{Submit your next manuscript to BioMed Central and take full advantage of:}

- Convenient online submission

- Thorough peer review

- No space constraints or color figure charges

- Immediate publication on acceptance

- Inclusion in PubMed, CAS, Scopus and Google Scholar

- Research which is freely available for redistribution 